\title{
A double complication may yield a positive outcome: air embolism in a huge aneurysm
}

\author{
Mostafa Dastani ${ }^{1}$, Nima Nakhai ${ }^{2}$, Majid Jalalyazdi ${ }^{3}$
}

${ }^{1}$ MD, Cardiologist, Department of Cardiology, Ghaem Hospital, School of Medicine, Mashhad University of Medical Sciences, Mashhad, Iran

${ }^{2}$ Resident of Cardiology, Department of Cardiology, Ghaem hospital, School of Medicine, Mashhad University of Medical Sciences, Mashhad, Iran

${ }^{3}$ MD, Cardiologist, Department of Cardiology, Imam Reza hospital, School of Medicine, Mashhad University of Medical Sciences, Mashhad, Iran

Type of article: Case report

\begin{abstract}
Coronary artery air embolization is a rare event leading to catastrophic hemodynamic compromise within seconds after introduction of air within the coronary arteries. The management of massive air embolism should be extremely quick so as to prevent cardiac compromise. In this report, we present a case of massive air embolism in the left anterior descending artery during coronary angiography in a 47-year-old female with a history of smoking and hyperlipidemia, who was admitted due to typical exertional chest pain despite analgesic administration. Coronary angiography was performed for the patient which revealed huge aneurysm of the left main artery and total occlusion of LAD artery and air embolism in the LAD and left main artery. Blood flow was attained by passing a catheter through the LAD occlusion. The preexisting coronary artery disease in this patient has led to success in the management of massive coronary emboli. Massive coronary artery emboli are life threatening and require urgent management, however, some patients might benefit from coexisting coronary pathologies as in this case.
\end{abstract}

Keywords: Coronary angiography; Air embolism; Coronary aneurysm

\section{Introduction}

The incidence of coronary artery air embolism during coronary artery interventions is rare and varies from 0.24 to 0.84 percent depending on the experience of the specialist in angioplasty (1). This unpredictable event may range from an asymptomatic event to even cardiovascular collapse (2). Management of air embolism requires immediate attention and intervention depending on the patient's clinical condition and the size of embolism $(3,4)$. Mechanical and pharmacological management are considered in clinically symptomatic patients (5). Underlying conditions or comorbidities might have a direct or inverse effect on the management strategies of coronary artery air embolism. In the present report, we discuss a case of coronary artery air embolism distributed in an aneurismal left main artery and left anterior descending artery (LAD) following coronary angiography. Surprisingly, because of chronic ischemia of LAD territory and retrograde filling from right circumflex artery (RCA), the patient did not show hyper acute hemodynamic disturbance, and the comorbidity improved the outcome of medical management.

\section{Case presentation}

\subsection{Clinical presentation}

A 47-year-old woman with a history of hyperlipidemia, smoking and chronic stable angina and a history of taking anti angina medication experienced typical exertional chest pain, which had been exacerbated despite medical antianginal treatment. With an indeterminate exercise test result, the patient, with stable hemodynamics, was admitted to an angiography ward for selective coronary angiography. The ETT was undetermined because the

\section{Corresponding author:}

Cardiology department, Ghaem hospital, Mashhad, Iran. Postal code: 9186953313.

Tel.: +98.9155072379, Fax: +98.5138022748, Email: nimanakhai@hotmail.com

Received: February 18, 2017, Accepted: November 23, 2017, Published: September 2019

iThenticate screening: October 04, 2017, English editing: May 12, 2018, Quality control: May 14, 2018

(C) 2019 The Authors. This is an open access article under the terms of the Creative Commons Attribution-NonCommercialNoDerivs License, which permits use and distribution in any medium, provided the original work is properly cited, the use is non-commercial and no modifications or adaptations are made. 
patient could not complete the ETT protocol and achieve maximum heart rate. According to her history of stable angina and symptoms refractory to drugs, we scheduled the patient for coronary angiography and revascularization.

\subsection{Angiography procedure}

A radial artery access was obtained, and left and right $3.5 \mathrm{~F}$ judkins diagnostic catheters were used. During engagement of left main coronary artery, guide wire 0.0035 was used by a third-year cardiology resident and interventional cardiology fellowship. After engagement of the left main coronary artery and testing for assurance of engagement, angiogram of left coronary system in RAO CAUDAL view was performed which showed huge aneurysm of the left main artery and total occlusion of the LAD artery from proximal, with clear cut off of several air bubbles in angiogram of previous LAD parts and left main artery. Left circumflex artery angiogram was normal (Figure 1). Meanwhile, the patient experienced chest pain, diaphoresis and ST segment elevation in precordial, I and avL leads with hyper acute $T$ wave. The patient's heart rate increased to $120 \mathrm{bpm}$ and blood pressure declined to 90/60 $\mathrm{mmHg}$ (patient primary blood pressure was $110 / 75 \mathrm{mmHg}$ ).

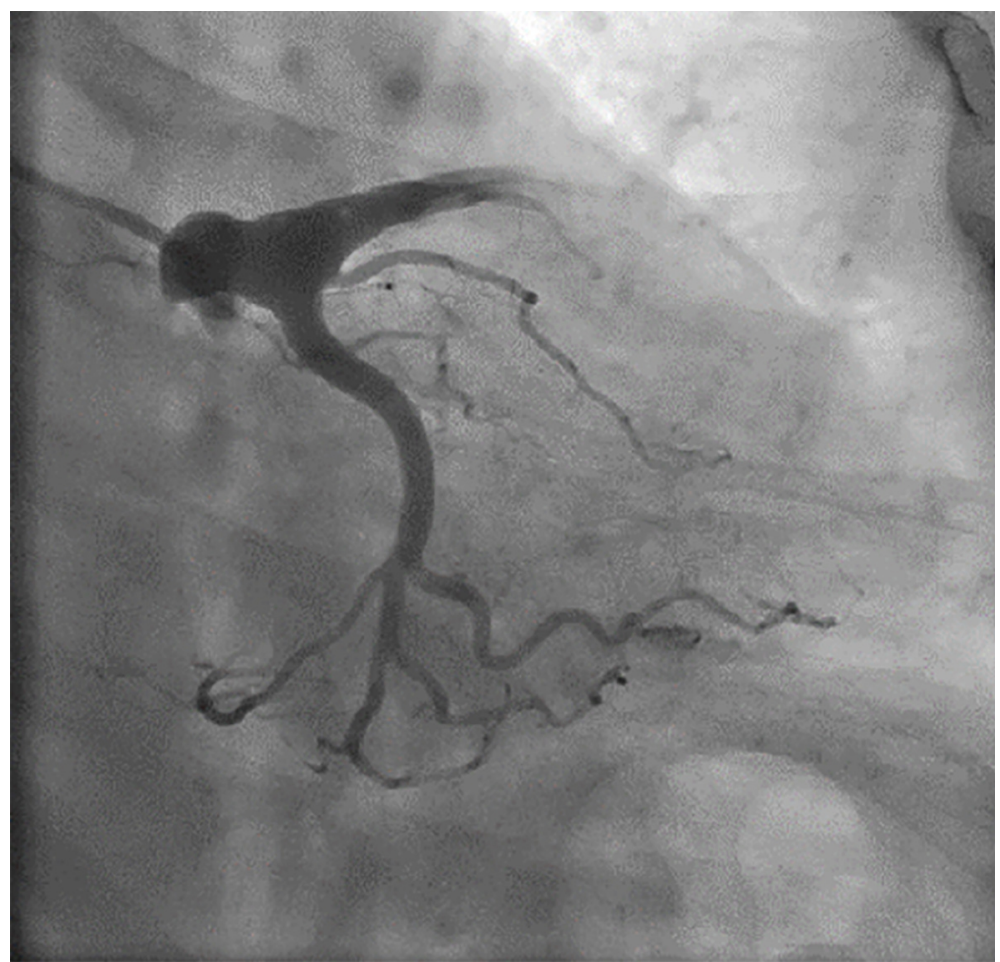

Figure 1. Angiogram shows left main artery aneurysm, left anterior descending artery total occlusion with air bubbles

\subsection{Treatment}

Quickly, oxygen therapy with facemask and hydration with normal saline serum was applied. Intravenous morphine was injected in order to reduce pain. Injection of saline in patient's coronary artery was done forcefully to dislodge the air bubble. Unfortunately, the attempt was not successful. Due to huge aneurysm of left main artery and suspicion of probable chronic occlusion of LAD and semi stable situation of patient, right coronary artery angiogram was done urgently. The result showed normal right coronary artery with retrograde filling of LAD (up to proximal part- rentrope 3), as seen in Figure 2. Again, left coronary angiogram was done which showed persistent cut off of the LAD artery. Urgently, a guiding catheter was applied and an angioplasty wire (BMW) was crossed through the LAD artery which restored flow to the LAD artery (TIMI3), probably due to bubbles bursting with wire passage. The patient's symptoms relieved dramatically and surface ECG ST segment elevation resolved within minutes of reperfusion. No obvious stenosis was seen in the LAD artery after reperfusion, and reason of retrograde filling of LAD artery via RCA was not clear in the perfusion scan (Figure 3). In respect of the patient's hemodynamic stability, the patient's RCA angiogram was obtained again, and showed retrograde filling of the LAD artery, weaker than the previous time (rentrope 2) as shown in Figure 4. 


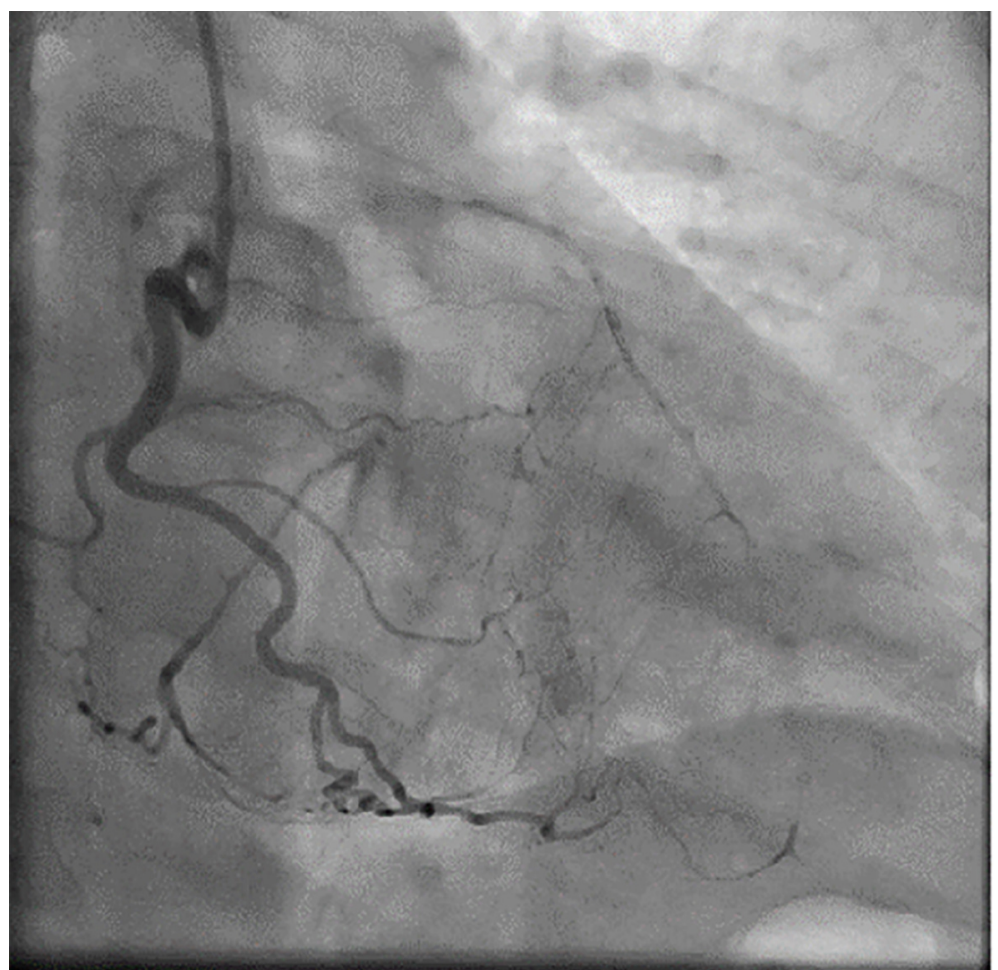

Figure 2. Angiogram shows retrograde filling of left anterior descending artery from right coronary artery.

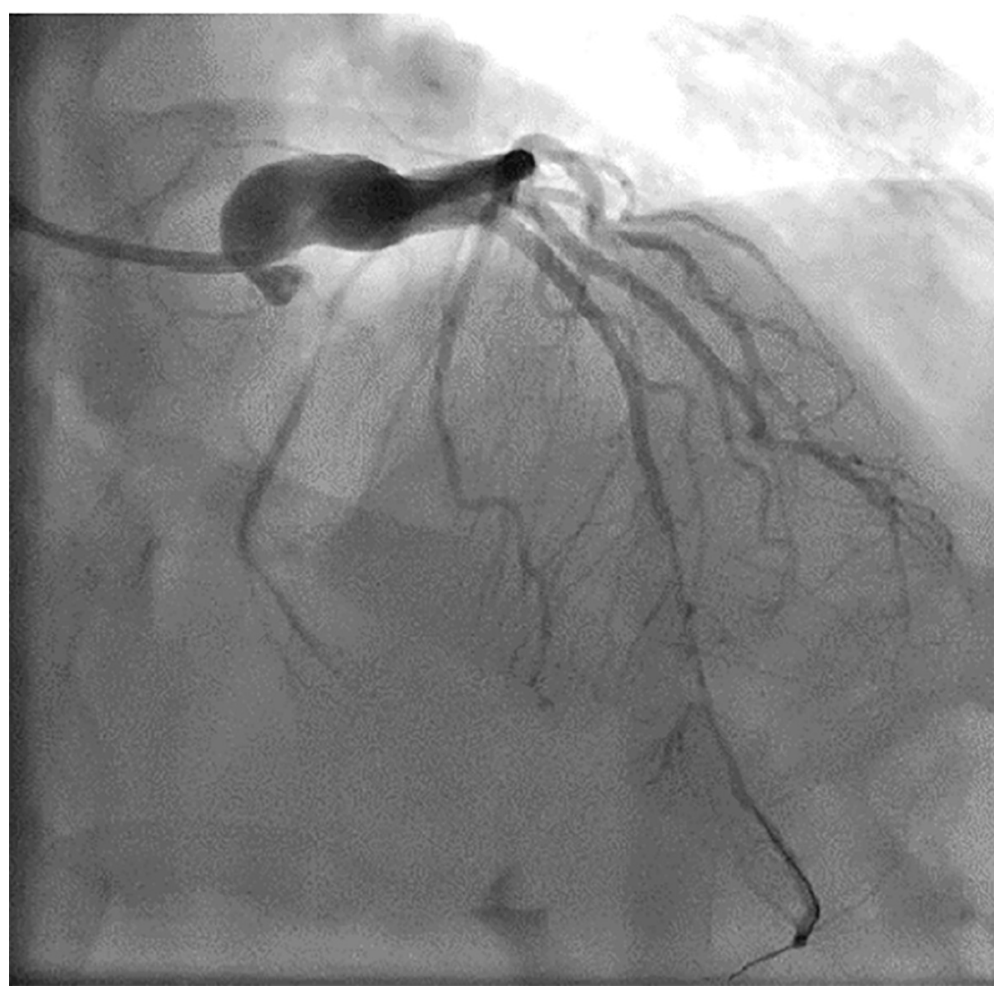

Figure 3. Successful restoration of flow in left anterior descending artery after passing angioplasty wire 


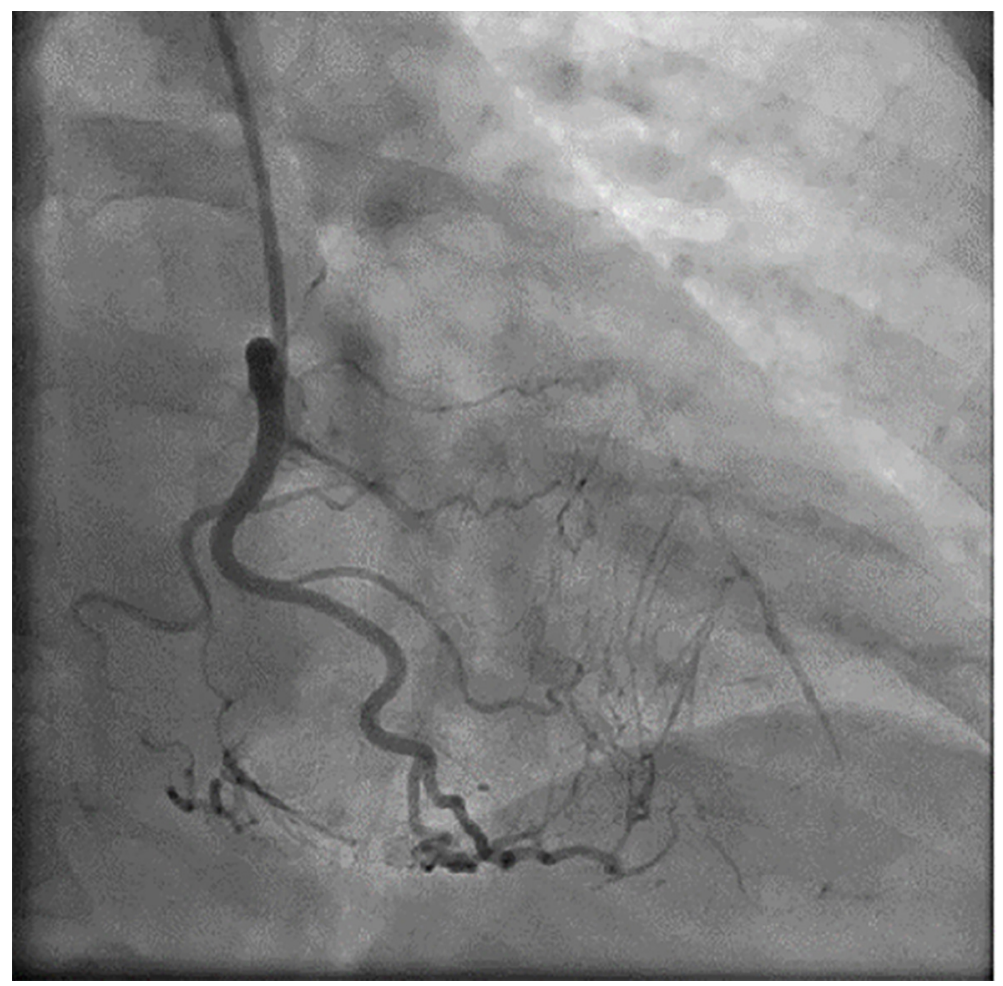

Figure 4. Reduced retrograde filling of left anterior descending artery after restoration of flow.

\subsection{Patient follow-up}

The patient was transferred from the catheterization laboratory to the cardiac care unit after 2 hours. We consulted with the cardiac surgeon for coronary artery bypass graft (CABG) due to LM aneurysm and electro and echocardiographic symptoms of Ischemia. According to the surgeon's opinion regarding the risk of CABG and the patient's unwillingness to undergo any cardiac surgery, we decide to intensify medical treatment. The patient was discharged after 7 days with isosorbide dinitrate 40mg TDS added to previous medications. After 6 months of follow up, there was no disabling symptom.

\subsection{Ethical consideration}

Written informed consent was obtained from the patient for publication of this case report and any accompanying images.

\section{Discussion}

While coronary air embolism is potentially a life-threatening event, our patient did not experience severe hemodynamic collapse or bradycardia and asystole, which have been seen in similar cases. This condition could be a result of preconditioning of the LAD territory due to chronic ischemia induced by slow flow secondary to huge left main artery aneurysm and retrograde filling via RCA with competitive flow which all together secured our patient from experiencing a much worse outcome. There are various ways for air entry to be introduced into the coronary circulation (2). The air can be resulted from incorrectly assembled equipment with loose connections or a partially de-aired manifold. The second way of air entrance may be caused by a Venturi-like effect during rapid withdrawal of the wire from the catheter prior to connecting to the manifold. The third reason can be a rupture of coronary dilatation catheters (2). The corner stone of successful management of air emboli is immediate diagnosis and prompt management. While micro bubbles may pass down the coronary three without producing remarkable symptoms, larger emboli can block coronary circulation, resulting in clinical symptoms. Depending on the patient's condition, prolonged cardiopulmonary resuscitation may be necessary (2). The first step in treating coronary embolism is administration of $100 \%$ oxygen. Bradycardia can be managed by intravenous atropine and transient increasing in arterial blood pressure can be achieved by using inotropic agents. Also, intracoronary adenosine and calcium channel blockers are useful in treating vasospasm. Air bubbles can be fragmented by forceful injection of contrast media or saline. Distal bubbles can be disrupted by use of guide wire $(2,4)$. In our case, while forceful saline injection was not successful in brushing the air bubble, wire passage was the fastest choice for bubble bursting, 
which was successful. Unlike the findings of this report, a previous case report revealed successful management of coronary artery air embolism by forceful infusion of contrast dye (3). Park et al. reported the case of a 65 -year-old female undergoing coronary angiography who developed air embolism in the left coronary artery because of incomplete aspiration of angiographic catheter (3). Their patient developed complete atrioventricular block and hypotension. The air bubbles were dispersed distally by forceful injection of contrast dye for several times, and their patient recovered from shock (3). In contrast to our case, in another case report coronary air embolism was resolved by saline flush. Chand et al. reported a case of coronary artery air embolism which was diagnosed by sudden chest pain, hypotension, loss of conciseness and sever bradycardia (4). They implanted a temporary transvenous pacemaker as well as administrating hyperbaric oxygen, dopamine, atropine and adrenaline. Multiple saline flush was used to dissipate the embolus (4). Patterson et al. reported a different management by performing Export aspiration catheter which resulted in successful resolution of the emboli (6).

\section{Conclusions}

In this report, the case of a 47-year-old female was presented with the complaint of exertional dyspnea and massive coronary air embolism due to angiography. We tried to brush the air bubbles with forceful saline injection and passing guide wire. However, in our case, the patient's previous preconditioning and retrograde filling of LAD via RCA was so much helpful and we were quite fortunate. Treatment of coronary air embolism has to be rapid and prompt as it was in our case, as well as providing 100\% oxygen, hydration and injecting analgesics.

\section{Acknowledgments:}

The authors wish to express their gratitude to the Imam Reza Hospital Cardiology Department for providing us the opportunity to access the medical records, and the Cardiac Surgery Department for their cooperation in the management of this patient.

\section{Conflict of Interest:}

There is no conflict of interest to be declared.

Authors' contributions:

All authors contributed to this project and article equally. All authors read and approved the final manuscript.

\section{References:}

1) Khan M, Schmidt DH, Bajwa T, Shalev Y. Coronary air embolism: incidence, severity, and suggested approaches to treatment. Catheterization and Cardiovascular Interventions. 1995; 36(4): 313-8. doi: 10.1002/ccd.1810360406. PMID: 8719380.

2) Kelly DJ. Coronary Air Embolism. Complications of Percutaneous Coronary Intervention. Springer. 2016: 145-50. doi: 10.1007/978-1-4471-4959-0_18

3) Park C, Hwang H, Cho J, Jo B, Kim C. Massive right coronary air embolism in the right coronary artery during left coronary angiography: A case report. Exp Ther Med. 2013; 5(4): 1073-4. doi: 10.3892/etm.2013.939. PMID: 23596473, PMCID: PMC3627689.

4) Chand P, Pandit N, Gajiwala N, Thakkar A. To air is human, to liberate is divine! Heart India. 2016; 4(2): 76. doi: 10.4103/2321-449X.183529.

5) Yew KL, Razali F. Massive coronary air embolism successfully treated with intracoronary catheter aspiration and intracoronary adenosine. Int J Cardiol. 2015; 188: 56-7. doi: 10.1016/j.ijcard.2015.04.040. PMID: 25885752.

6) Patterson MS, Kiemeneij F. Coronary air embolism treated with aspiration catheter. Heart. 2005; 91(5): e36. doi: 10.1136/hrt.2005.060129. PMID: 15831621, PMCID: PMC1768886. 